\title{
Minimization of Call Blocking Probability using Mobile Node velocity
}

\author{
Suman Kumar Sikdar \\ Department of Computer science \& \\ Engineering, University of Kalyani \\ Kalyani-741235, India
}

\author{
Uttam Kumar Kundu \\ MTech in Department of Electronics \\ \& Communication Engineering, W.B. \\ University of Technology, India
}

\author{
Debabrata Sarddar (Astt \\ Professor) \\ Department of Computer Science\& \\ Engineering, University of kalyani \\ Kalyani-741235
}

\begin{abstract}
Due to rapid growth in IEEE 802.11 based Wireless Local Area Networks (WLAN), handoff has become a burning issue. A mobile Node (MN) requires handoff when it travels out of the coverage area of its current access point (AP) and tries to associate with another AP. But handoff delays provide a serious barrier for such services to be made available to mobile platforms. Throughout the last few years there has been plenty of research aimed towards reducing the handoff delay incurred in the various levels of wireless communication.In this article, we propose a received signal strength measurement based handoff technique to improve handoff probability. By calculating the speed of MN (Mobile Node) and signaling delay information we try to take the right decision of handoff initiation time. Our theoretical analysis and simulation results show that by taking the proper decision for handoff we can effectively reduce false handoff initiation probability and unnecessary traffic load causing packet loss and call blocking.
\end{abstract}

Keywords-component; Next Generation Wireless Systems (NGWS); Handoff; BS (Base Station); MN (Mobile Node); RSS (Received signal strength); IEEE802.11

\section{INTRODUCTION}

Handoff has become an essential criterion in mobile communication system especially in urban areas, owing to the limited coverage area of Access Points (AP). Whenever a MN move from current AP to a new AP it requires handoff. For successful implementation of seamless Voice over IP communications, the handoff latency should not exceed 50ms. But measurements indicate MAC layer handoff latencies in the range of $400 \mathrm{~ms}$ which is completely unacceptable and thus must be reduced for wireless networking to fulfil its potential.

With the advent of real time applications, the latency and packet loss caused by mobility became an important issue in Mobile Networks. The most relevant topic of discussion is to reduce the IEEE 802.11 link-layer handoff latency. IEEE 802.11 MAC specification [1] defines two operation modes: ad hoc and infrastructure mode. In the ad hoc mode, two or more stations (STAs) recognize each other through beacons and hence establish a peer-to-peer relationship. In infrastructure mode, an AP provides network connectivity to its associated STAs to form a Basic Service Set (BSS).

Multiple APs form an Extended Service Set (ESS) that constructs the same wireless networks.

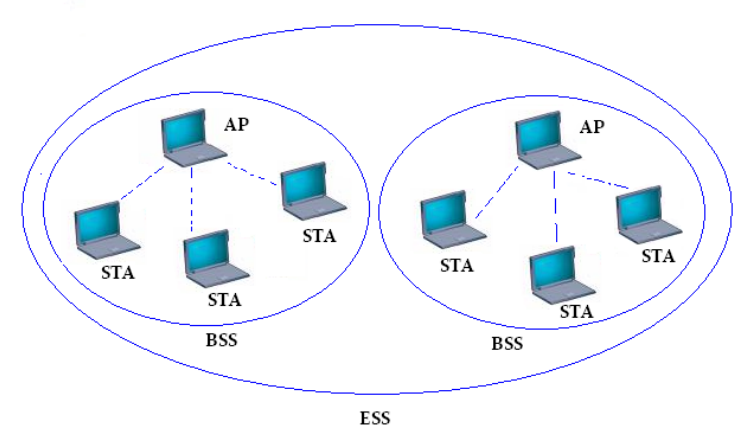

Figure1.

\section{A. Channel distribution}

IEEE $802.11 \mathrm{~b}$ and IEEE $802.11 \mathrm{~g}$ operates in the $2.4 \mathrm{GHz}$ ISM band and use 11 of the maximum 14 channels available and are hence compatible due to use of same frequency channels. The channels (numbered 1to14) are spaced by $5 \mathrm{MHz}$ with a bandwidth of $22 \mathrm{MHz}, 11 \mathrm{MHz}$ above and below the centre of the channel. In addition there is a guard band of $1 \mathrm{MHz}$ at the base to accommodate out-of-band emissions below $2.4 \mathrm{GHz}$. Thus a transmitter set at channel one transmits signal from $2.401 \mathrm{GHz}$ to $2.423 \mathrm{GHz}$ and so on to give the standard channel frequency distribution as shown in [Figure.2]

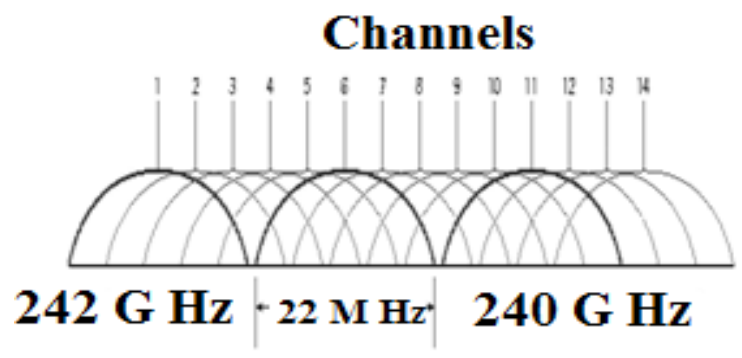

Figure 2 .

It should be noted that due to overlapping of frequencies there can be significant interference between adjacent APs. Thus, in a well configured network, most of the APs will operate on the non-overlapping channels numbered 1, 6 and 11. 


\section{B. Handoff:}

When a MS moves out of reach of its current AP it must be reconnected to a new AP to continue its operation. The search for a new AP and subsequent registration under it constitute the handoff process which takes enough time (called handoff latency) to interfere with proper functioning of many applications

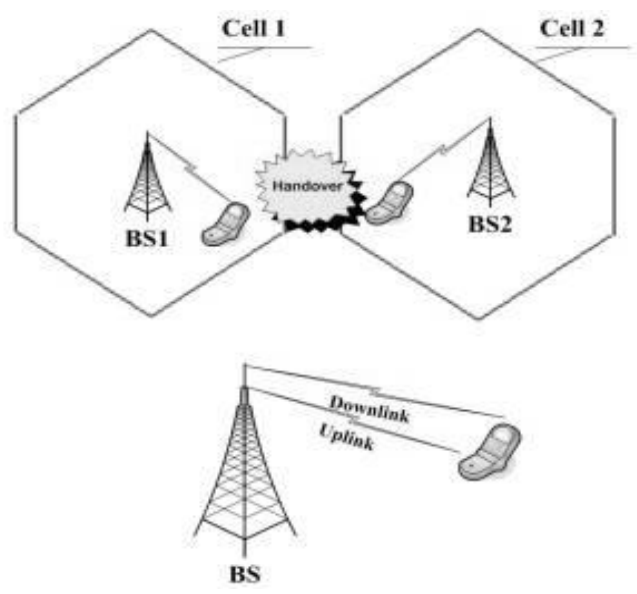

Figure 2 : Handoff process

Three strategies have been proposed to detect the need for hand off[1]:

1) mobile-controlled-handoff (MCHO):The mobile station(MS) continuously monitors the signals of the surrounding base stations(BS)and initiates the hand off process when some handoff criteria are met.

2) network-controlled-handoff (NCHO):The surrounding $B S s$ measure the signal from the MS and the network initiates the handoff process when some handoff criteria are met.

3) mobile-assisted-handoff (MAHO):The network asks the $M S$ to measure the signal from the surrounding BSs. The network makes the handoff decision based on reports from the $M S$.

Handoff can be of many types:

Hard \& soft handoff: Originally hard handoff was used where a station must break connection with the old AP before joining the new AP thus resulting in large handoff delays. However, in soft handoff the old connection is maintained until a new one is established thus significantly reducing packet loss.

The handoff procedure consists of three logical phases where all communication between the mobile station undergoing handoff and the APs concerned is controlled by the use of IEEE802.11 management frames as shown below in [fig3].

\section{Scanning:}

When a mobile station is moving away from its current $\mathrm{AP}$, it initiates the handoff process when the received signal strength and the signal-to-noise-ratio have decreased significantly. The STA now begins MAC layer scanning to find new APs. It can either opt for a passive scan (where it listens for beacon frames periodically sent out by APs) or chose a faster active scanning mechanism wherein it regularly sends out probe request frames and waits for responses for $\mathrm{T}_{\mathrm{MIN}}$ (min Channel Time) and continues scanning until $\mathrm{T}_{\mathrm{MAX}}$ (max Channel Time) if at least one response has been heard within $\mathrm{T}_{\text {MIN. }}$. Thus, $\mathrm{n}^{*} \mathrm{~T}_{\mathrm{MIN}} \leq$ time to scan $\mathrm{n}$ channels $\leq$ $\mathrm{n}^{*} \mathrm{~T}_{\text {MAX }}$. The information gathered is then processed so that the STA can decide which AP to join next. The total time required until this point constitutes $90 \%$ of the handoff delay.

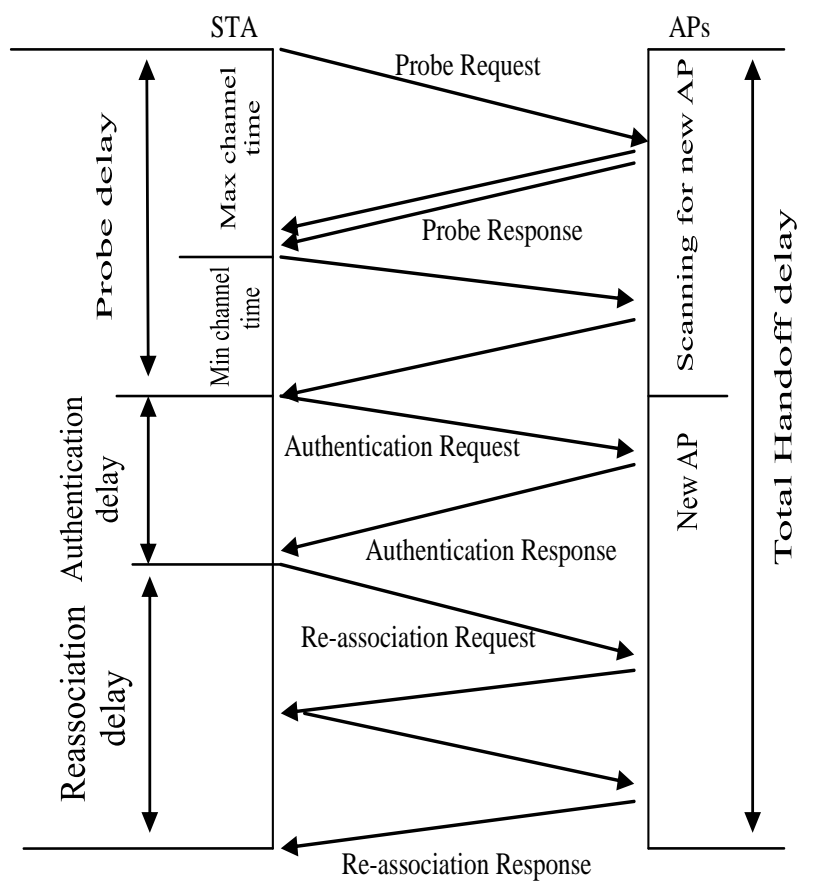

Figure 3

\section{Authentication:}

Authentication is necessary to associate the link with the new AP. Authentication must either immediately proceed to association or must immediately follow a channel scan cycle. In pre-authentication schemes, the $\mathrm{MN}$ authenticates with the new AP immediately after the scan cycle finishes. IEEE 802.11 defines two subtypes of authentication service: 'Open System' which is a null authentication algorithm and 'Shared Key' which is a four-way authentication mechanism. If Inter Access Point Protocol (IAPP) is used, only null authentication frames need to be exchanged in the re-authentication phase. Exchanging null authentication frames takes about 1-2 ms.

\section{E. Re-Association:}

Re-association is a process for transferring associations from old AP to new one. Once the STA has been authenticated with the new AP, re-association can be started. Previous works has shown re-association delay to be around 1-2 ms. The range of scanning delay is given by:- 
$\mathrm{N} \times \mathrm{Tmin}_{\text {_ }}$ Tscan _ $\mathrm{N} \times \mathrm{Tmax}$

Where $\mathrm{N}$ is the total number of channels according to the spectrum released by a country, Tmin is Min Channel Time,Tscan is the total measured scanning delay, and Tmax is Max Channel Time. Here we focus on reducing the scanning delay by minimizing the total number of scans performed.

We have organized the rest part of this paper as follows. Sec 2 describes the related works, Sec 3 describes our proposed approach. The simulation results are given in Sec 4 and finally concluding remarks are presented in Sec5.

\section{RELATED WORKS}

A lot of researches have been dedicated to enhance the performance of handover in Next Generation heterogeneous Wireless Networks. Recently a number of cross layer protocols and algorithms have been proposed to support seamless handoff in NGWS.

A number of different schemes have been proposed to reduce handoff latency in IEEE 802.11 wireless LANs. IEEE 802.11b based wireless and mobile networks [5], also called Wi-Fi commercially, are experiencing a very fast growth upsurge and are being widely deployed for providing variety of services as it is cheap, and allows anytime, anywhere access to network data.

The new age applications require a seamless handover while the small coverage of individual APs has increased the number of handoffs taking place. Thus reducing the handoff latency has become a burning issue and much work has been done to achieve this. See [6] for an overall review of popular methods suggested.

Shin et al in [7] have introduced a selective scanning algorithm with the help of channel masking technique coupled with a caching mechanism to significantly reduce the handoff delay. However, it still scans excess APs even after the new AP may have already been found and thus leaves room for further improvements.

Handoff, an inherent problem with wireless networks, particularly real time applications, has not been well addressed in IEEE 802.11, which takes a hard handoff approach [8].

In [9] the authors have introduced a novel caching process using neighbor graphs by pre-scanning neighbor APs to collect their respective channel information. The concept of neighbor graphs can be utilized in different ways and have become very popular in this field. In [10] a pre-authentication mechanism is introduced to facilitate seamless handover. [11] is a novel approach towards reducing handover latency in AP dense networks.

In [12] a cross layer handoff management protocol scheme has been proposed. They tried to enhance the handoff performance by analyzing the speed of the mobile node, handoff signaling delay, relative signal strength of old and new base station and their relation with handoff failure probability.

A novel mobility management system is proposed in [13] for vertical handoff between WWAN and WLAN. The system integrates a connection manager (CM) that intelligently detects the changes in wireless network and a virtual connectivity manager (VCM) mantains connectivity using end-to-end priciple.

Authors of [14] propose solutions towards enabling and supporting all types of mobility in heterogeneous networks. The proposed approach does not support real time applications by the network mobility functionality. This keeps the application unaware of network mobility and works as a backup for real time applications.

Handoff using received signal strength (RSS) of BS has been proposed also to reduce handoff latency in NGWS.

In [15], the authors proposed a handoff algorithm in which the received pilot signal strength is typically averaged to diminish the undesirable effect of the fast fading component. Unfortunately, the averaging process can substantially alter the characteristics of path loss and shadowing components, causing increased handoff delay.

In [16], a handoff algorithm using multi-level thresholds is proposed. The performance results obtained, shows that an 8level threshold algorithm operates better than a single threshold algorithm in terms of forced termination and call blocking probabilities.

In [17] signal to interference ratio between old base-station and neighboring base-stations are calculated to make the handoff initiation decision for next generation wireless system or $4 \mathrm{G}$ networks.

In [18], a handoff algorithm using multi-level thresholds is proposed. The performance results obtained, shows that an 8-level threshold algorithm operates better than a single threshold algorithm in terms offorced termination and call blocking probabilities.

In [23], a handoff algorithm using distance measurement method is used where the distance of MN from each BS is calculated and by comparing them the best neighbor AP can be found out.

In [24], a handoff algorithm using cell sectoring method is used where the hexagonal cell is divided in 3 sectors and each sector is assigns with two neighbor APs.

In [25], a handoff algorithm using prescanning technique is used where the neighbor graphs are used to decide the handoff. In [26], carrier to interference ratio is calculated to find the best neighbor AP to perform handoff.

A new handoff scheme is proposed in [27] where a curve fitting equation is used to predict the direction of motion of $\mathrm{MN}$ and thus perform the handoff by reducing the scanning time in a considerable amount.

In [28] and [29] two schemes are proposed to reduce handoff failure probability by introducing new cell coverage area in handoff region and by using different channel allocation technique.

In [30] a vector analysis method is used to reduce the handoff latency where the direction of velocity of $\mathrm{MN}$ is considered as a vector quantity. 
Another new handoff scheme is proposed in [31] where a tangent analysis method is used to predict the direction of motion of MN and thus perform the handoff by reducing the scanning time in a considerable amount.

\section{PROPOSED WORK}

In this paper we propose a handoff management protocol to support seamless handoff in Next Generation Wireless Systems. We consider the mobile node's speed, Relative Signal Strength of the base station, handoff signaling delay information and threshold distance from cell boundary to reduce false handoff initiation probability which creates unnecessary traffic load and sometimes call blocking.

For our proposed work we consider the coverage area of the base stations (BS) as regular hexagonal cells. We take two base stations into our account to explain our proposed approach, one is OBS where the call generates and other is $\mathrm{NBS}$, next destination of the MN. When the MN tends to move out the coverage area of OBS it needs handoff with NBS to continue the call. Fig. 4 describes the handoff scenario between OBS nad NBS. Notations used in Fig. 4 describes the following parameters.

$S_{t h}=$ The threshold value of the RSS to initiate the handoff process. When the RSS of old BS (OBS) referred to as ORSS drops below $\boldsymbol{S}_{\boldsymbol{t} \boldsymbol{h}}$ the MN starts the HMIP registration procedures for handoff to new BS (NBS).

$S_{\min }=$ The minimum value of RSS required for successful communication between the MN and OBS.

$a=$ The cell size.

$d=$ The threshold distance from cell (OBS) boundary, where the MN starts HMIP registration.

We divide our proposed work into four sections:

1) Speed Estimation.

2) Measurement of threshold distance dfrom the cell (OBS) boundary.

3) RSS and $\boldsymbol{S}_{\boldsymbol{t h}}$ Measurement.

4) Handoff Management.

B. Speed Estimation:

The speed of the $\mathrm{MN}$ is estimated by using the Doppler Effect. The received signal frequency and the carrier signal frequency and the speed of the MN are respectively $f_{r}, f_{c}$, and $v_{m}$. And $\mathrm{v}_{\mathrm{c}}$ is speed of the light. Hence

$$
\begin{aligned}
& f_{r}=\frac{\left(v_{c}-v_{m} \cos \theta\right)}{v_{c}} \cdot f_{c} \ldots \ldots \ldots \ldots \ldots \ldots \ldots \\
& \text { or }, \quad f_{r} / f_{c}=\left(1-\frac{v_{m}}{v_{c}} \cdot \cos \theta\right)
\end{aligned}
$$

www.ijacsa.thesai.org

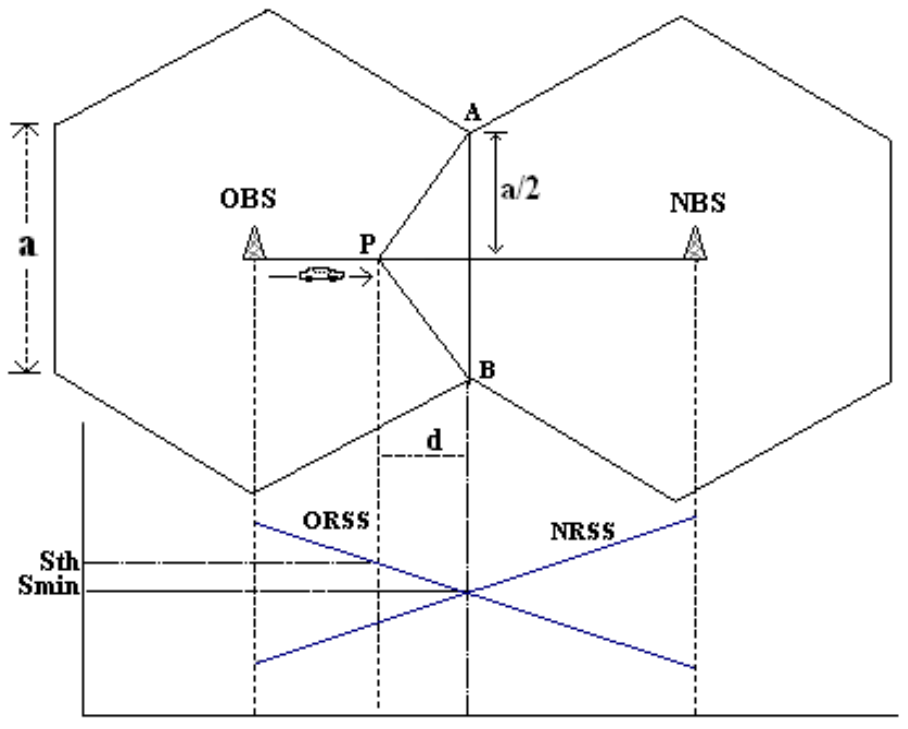

Figure 4

$$
\begin{aligned}
\text { or, } & v_{m}=\frac{\left(f_{c}-f_{r}\right)}{f_{c}} \cdot \sec \theta \\
\text { or, } \quad v_{m} & =\left(1-\frac{f_{r}}{f_{c}}\right) \cdot v_{c} \sec \theta
\end{aligned}
$$

This equation helps to estimate the speed of the MN.

When the MN moves out of the coverage area of OBS through radial outward direction, $\sec \theta=1$.

Thus

$$
v_{m}=\left(1-\frac{f_{r}}{f_{c}}\right) \cdot v_{c}
$$

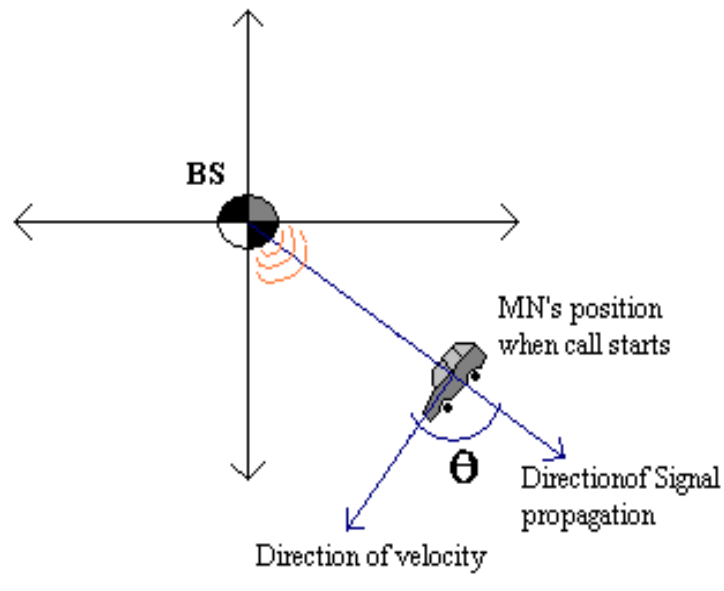

Figure 5 


\section{Measurement of threshold distance d from the cell}

(OBS) boundary:

Case1: Let handoff signaling delay is $\Gamma$, when the $\mathrm{MN}$ moves out of the coverage area of OBS through radically outward (Fig.5) direction the MN needs to initiate HMIP registration at the distance $d$ from the cell boundary for a successful handoff. Otherwise the MN does not get enough time to complete the total handoff procedure.

So for a successful handoff (handoff success probability maximum) in this case

$$
d \leq \Gamma . v_{m}
$$

Case2: When the MN reaches the point P, the RSS starts to drop below the $\boldsymbol{S}_{\boldsymbol{t} \boldsymbol{h}}$ value. Here the MN needs to initiate HMIP registration. Now the $\mathrm{MN}$ can move to any direction from $\mathrm{P}$ with equal probability. For a worst case if MN moves out through PA or PB (Fig. 4), it remains within the $\boldsymbol{S}_{\boldsymbol{t h}}$ region and overlap region of OBS and NBS. So the MN can initiate handoff without any hesitation from here. In this case

$$
\begin{array}{r}
\begin{array}{r}
\mathrm{PA}=\mathrm{PB}=\sqrt{(\mathrm{a} / 2)^{2}+\mathrm{d}^{2}} \\
\text { For handoff }
\end{array} \\
\Gamma<\frac{\sqrt{(a / 2)^{2}+d^{2}}}{v_{m}} \\
\text { or, } d>\sqrt{\left(\Gamma \cdot v_{m}\right)^{2}-a^{2} / 4} \ldots \ldots \ldots
\end{array}
$$

In this case handoff failure probability is maximum.

Combining (3) and (4) we get,

$$
\sqrt{\left(\Gamma \cdot v_{m}\right)^{2}-a^{2} / 4}<d \leq \Gamma . v_{m}
$$

For this condition handoff success probability lies between zero to one.

\section{RSS and $\boldsymbol{S}_{\text {th }}$ Measurement:}

The OBS antenna is transmitting a signal which gets weaker as The MN moves away from it. The transmitted signal power is maximum at the centre of the cell and gradually decreases as the distance from the centre increases. The received signal strength (RSS) at a distance $\boldsymbol{x}$ is given by

$$
P_{r}=\frac{G_{r} \cdot G_{t} \cdot P_{t}}{\left(\frac{4 \Pi x}{\lambda}\right)^{2}}
$$

$\lambda=$ wave length of the transmitting signal.

$\mathrm{G}_{\mathrm{r}}=$ Receiving antenna gain.

$\mathrm{G}_{\mathrm{t}}=$ Transmitting antenna gain. $P_{t}=$ Transmitting power.

The threshold value of the received signal strength,

$$
S_{t h}=\frac{G_{r} \cdot G_{t} \cdot P_{t}}{\left(\frac{4 \Pi y}{\lambda}\right)^{2}}
$$

$$
\begin{aligned}
& \text { Where } y=\left(\frac{\sqrt{3}}{2} a-d\right) \\
& \text { And } d=\Gamma \cdot v_{m}
\end{aligned}
$$

We take this value as we calculate the limiting value for $d$ previously. After this distance the MN can starts handoff initiation. After call setup the MN periodically checks the RSS, when RSS drops below the calculated threshold value the MN tries to initiate HMIP registration.

The wave propagation in multipath channel depends on the actual environment, including factors such as the antenna height, profiles of buildings, roads and geo-morphological conditions (hills, terrain) etc. These factors cause propagation loss in the channel. Hence the received signal strength may be

$$
P_{r}=\frac{G_{r} \cdot G_{t} \cdot P_{t}}{L}
$$

Propagation loss $L$ is characterized by three aspects: path loss $\left(\boldsymbol{L}_{\boldsymbol{p}}\right)$, slow fading $\left(\boldsymbol{L}_{\boldsymbol{s}}\right)$ and fast fading $\left(\boldsymbol{L}_{\boldsymbol{f}}\right)$.

$$
L=L_{p} \cdot L_{s} \cdot L_{f}
$$

In a typical urban area propagation loss is measured as [11]

$$
\begin{gathered}
L_{p u}(\mathrm{~dB})=69.55+26.16 \log _{10} f_{c}(\mathrm{MHz}) \\
-13.82 \log _{10} h_{b}(\mathrm{~m})-\alpha\left[h_{m}(\mathrm{~m})\right] \\
+\left[44.9-6.55 \log _{10} h_{b}(\mathrm{~m})\right] \log _{10} x(\mathrm{~km}) \\
h_{b}=\text { Base antenna height } \\
h_{m}=\text { MN's antenna height }
\end{gathered}
$$

$x=$ Distance of MN from the BS. This can be calculated easily. By using Neighbor Graph, the position of MS $\left(\mathrm{x}_{\mathrm{m}}, \mathrm{y}_{\mathrm{m}}\right)$ and the position of $\mathrm{BS}\left(\mathrm{x}_{\mathrm{b}}, \mathrm{y}_{\mathrm{b}}\right)$ can be found out and from these two coordinate we can calculate the distance of MS from BS. So, the distance is:

$$
X=\sqrt{ }\left\{\left(x_{b}-x_{m}\right)^{2}+\left(y_{b}-y_{m}\right)^{2}\right\}
$$

$\alpha\left[h_{m}(m)\right]=$ correlation factor for the MN's antenna height. Where,

$$
\begin{gathered}
\alpha\left[h_{m}(m)\right]=\left[1.1 \log _{10} f_{c}-0.7\right] h_{m}(m)-\left[1.56 \log _{10} f_{c}\right. \\
-0.8]
\end{gathered}
$$

\section{E. Handoff Management:}

Handoff Initiation: When the MN is going to move out its old BS its first challenge is to estimate the right time to initiate the HMIP registration. The handoff trigger unit (Fig 6) estimates the speed of the $\mathrm{MN}$ and its direction of motion and signaling delay information to determine the threshold RSS $\left(\boldsymbol{S}_{\boldsymbol{t h}}\right)$. When the RSS drops below $S_{\text {th }}$ the handoff trigger unit sends a handoff request to handoff execution unit to start HMIP handoff procedures.

Handoff Execution:After receiving the handoff request from handoff trigger unit the MN starts HMIP registration. Once it is completed the MN switched to the new BS. The MN keeps its HMIP registration with its old BS for a specified time. This is implemented by simultaneous binding option of HMIP protocol. The MN binds care of address $(\mathrm{CoA})$ of the old foreign agent (OFA) and new FA (NFA) at the gateway 
FA in case of intrasystem handoff and at the HA in intersystem handoff. Therefore, the GFA and HA forward packets destined for the MN to both the CoAs during this time interval. It may be noted that for an intersystem handoff these two CoAs may belong to two different network interfaces when the MN moves between networks of different wireless technologies. Therefore multiple interfaces of the MN can be used to reduce the ping-pong effect during an intersystem handoff.

\section{Simulation Result}

In this section we evaluate the performance of our approach. For our simulation we consider a macro-cellular systems with a cell size of $\mathrm{a}=1 \mathrm{~km}$ and the maximum speed of the $\mathrm{MN}$ is $50 \mathrm{~km} /$ hour. We consider the sgnalling delay is 40 second and wavelength $(\lambda)$ is $.1 \mathrm{~m}$.

Here we consider an arbitrary movement of MS as shown in [figure7]. The green line in the figure shows the trajectory path of the MS. Now we will follow our proposed algorithm.

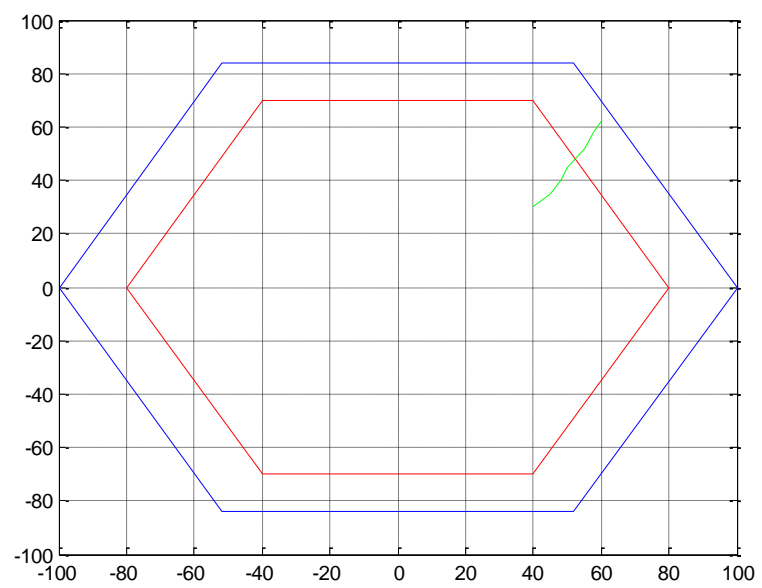

Figure $7(* 10)$

The relation between distance (from the center of the cell) covered by the $\mathrm{MN}$ and time is shown in figure 8 . Using equation (5), we can find out the range of threshold distance (d), as shown in figure 8. Again, Fig 9 shows the relation between MN's speed $(\mathrm{km} / \mathrm{h})$, MN's direction of motion (in Degree) from the place where the call was originated and the received signal frequency. The relation is established from the equation (1) using Doppler frequency shift method. The simulation result in $3 \mathrm{D}$ plot shows that by measuring the received signal frequency and the MN's direction of motion at different instant we can determine the MN's speed with which it is approaching towards the cell boundary.

We analyze the relationship between distance (in meter) from the Base Station and the Received Signal Strength (RSS) (in $\mathrm{dBm}$ ) in Fig.9 using the equation (6). In figure 10, the Received Signal Strength (RSS) (in $\mathrm{dBm}$ ) vs time (second) is shown. As the time increases, the distance of MN from the BS is increases and the RSS decreases exponentially.

After 7 seconds $(t=7)$, the Received Signal Strength (RSS) goes below threshold value and handoff initiated. We simulate the relation for three different values of carrier frequency using the parameters in Table1. This is shown in figure 11.

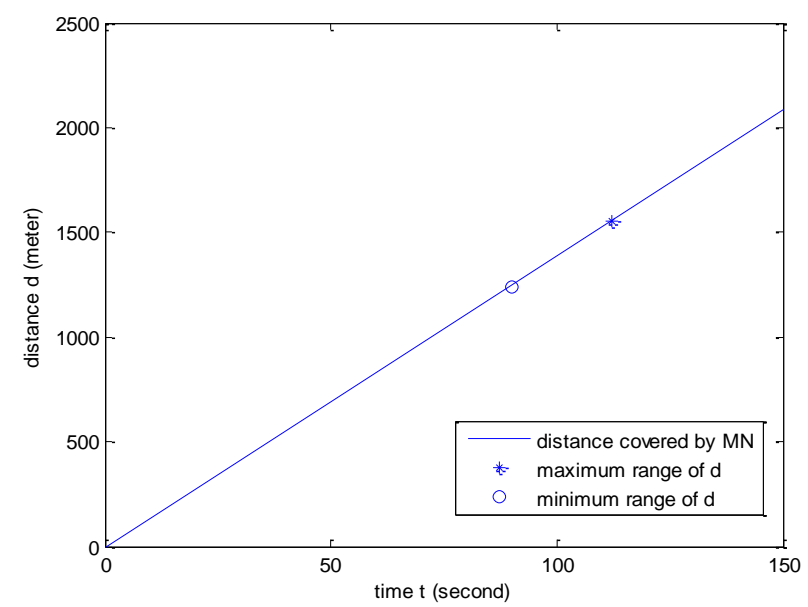

Figure 8

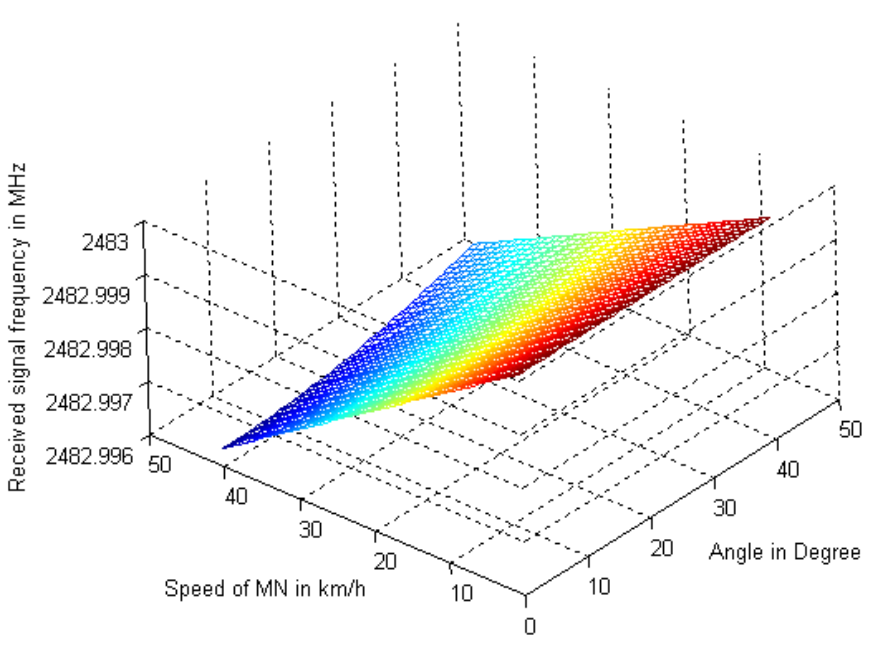

Figure 9

Table 1

\begin{tabular}{|c|}
\hline $\begin{array}{c}\text { Base Station antenna specification( Fiberglass } \\
\text { Rodome, Base Station Antenna) }\end{array}$ \\
\hline Frequency Range : $2.4-2.483 \mathrm{GHz}$ \\
\hline Gain: $18 \mathrm{dBi}$. \\
\hline Max input Power/Transmitter power: $115 \mathrm{dBm} / 35 \mathrm{dBm}$ \\
\hline Antenna height: $30 \mathrm{~m}$ \\
\hline Polarization: vertical \\
\hline $\begin{array}{c}\text { Cell phone antenna specification }(\mathbf{2 . 4 G H z} \text { Omni } \\
\text { Directional wireless antenna) }\end{array}$ \\
\hline Frequency Range : $2.4-2.483 \mathrm{GHz}$ \\
\hline Gain: $7 \mathrm{dBi}$. \\
\hline Max Power: $43 \mathrm{dBm}$ \\
\hline Antenna height: $1.6 \mathrm{~m}$ \\
\hline Polarization: vertical
\end{tabular}

Figure 10 


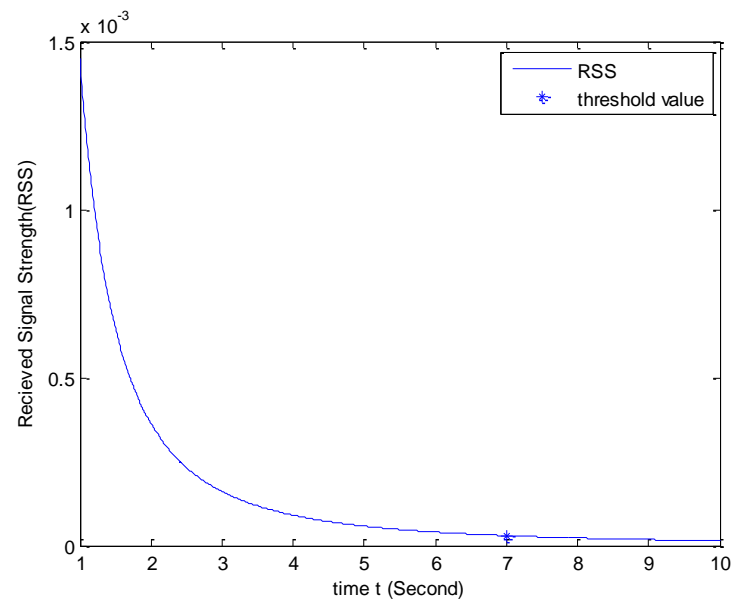

Figure 11

Fig 12 shows the relation between path-loss (in $\mathrm{dB}$ ) and the distance from the BS (in meter). Path-loss increases exponentially as the distance increases. Results in Fig 4 and Fig 12 are correlated, they describe that when distance from the BS increases path-loss increases exponentially and as a result RSS decreases.

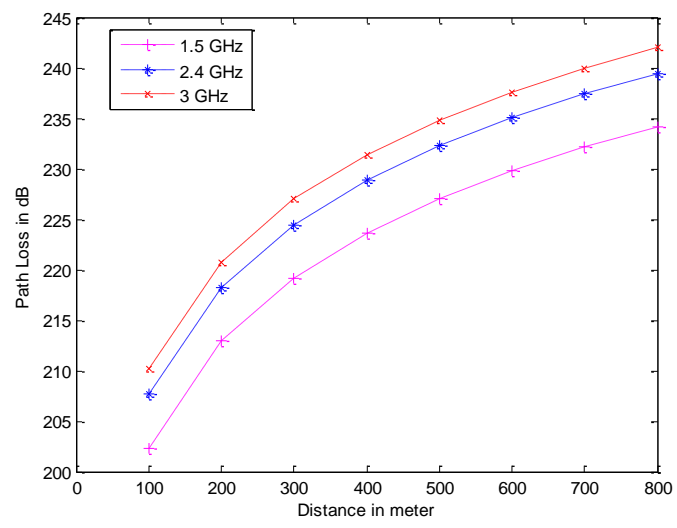

Figure 12

In figure 13, we analyze the relationship between time (in seconds) and Received Signal Strength (RSS) for three different values of MN velocity (30km/hr,50 km/hr,90 km/hr). MN with velocity $90 \mathrm{~km} / \mathrm{hr}$ crosses the threshold value at about 1.8 second, whereas $\mathrm{MN}$ with velocity $50 \mathrm{~km} / \mathrm{hr}$ crosses the threshold value at 7 second and MN with velocity 30 $\mathrm{km} / \mathrm{hr}$ crosses the threshold value after our observation time (10 seconds). It indicates that MN with higher velocity will require handoff earlier because it loses its received signal strength more rapidly.

Depending upon the level of traffic impediments we noted down the corresponding call blocking probability, that is the number of calls that terminates during handoff to the total number of handoffs in that traffic condition. The traffic of the network is taken relative to the maximum traffic.

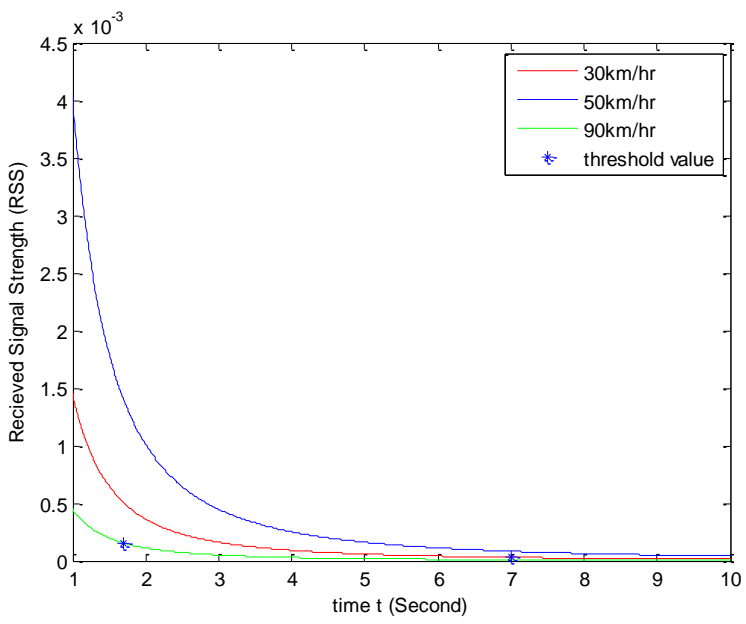

Figure 13

The plot of network traffic verses the call blocking probability is shown in the Figure 14 . We have also shown the handoff call blocking probability that is the number of calls that terminates during handoff to the total number of handoffs in that traffic condition, in the same base parameter in Figure 15.

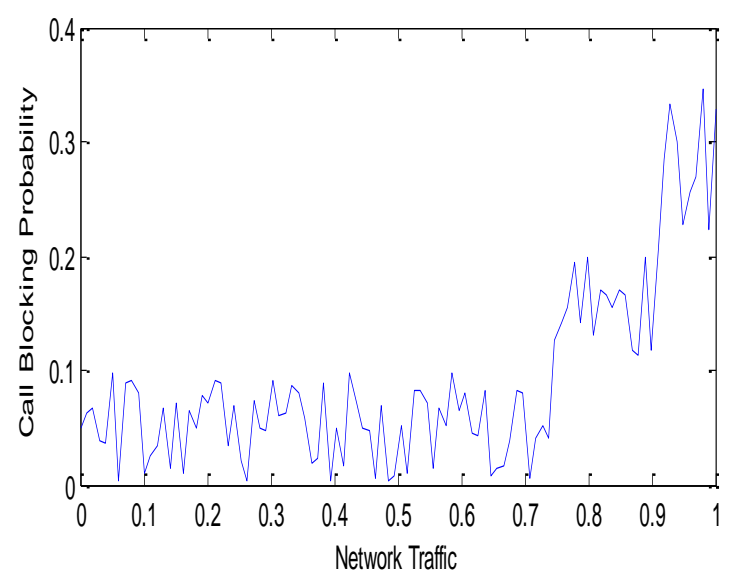

Figure 14. Plot of network traffic verses the call blocking probability

From Figure 14 it can be seen that the call blocking probability is below 0.1 for cases up to where sixty percent of the network is congested with a gradual increase, which is obvious for high congestion in the network. Figure 15 shows the significant improvement of handoff failure management against the conventional one by applying the new algorithm. Though handoff call blocking probability increases in an exponential manner with network traffic but we are able to restrict it to $8 \%$ of the maximum value which is a significant improve over the previous results. Thus our method effectively reduces the traffic blocking probability and also handoff call blocking probability.

\section{ACKNOWLEDGMENT}

In our article we discuss the different types of handoff procedures and associated problems for Next Generation Wireless Systems (NGWS). Then we describe our proposed 
handoff management using mobile node's (MN) speed, signaling delay information and RSS.

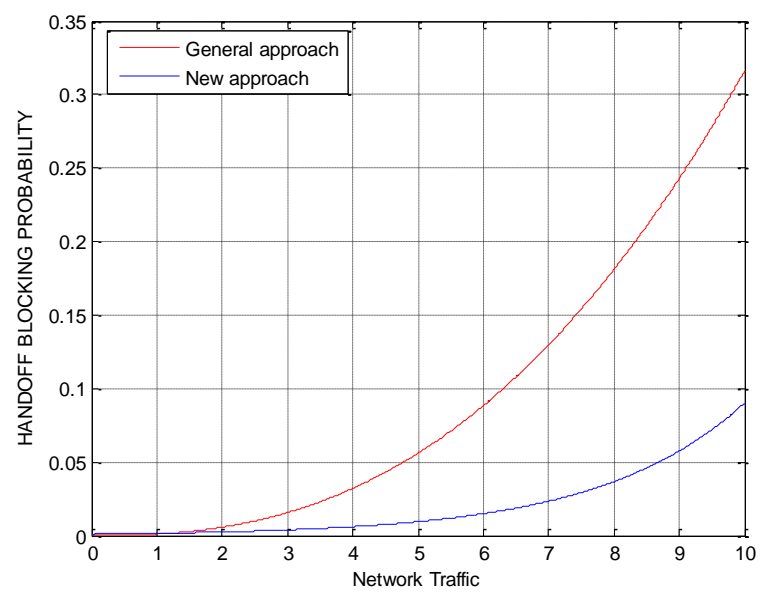

Figure 15. Plot of network traffic verses the handoff call blocking probability

We calculate the threshold value of RSS $\left(S_{t h}\right)$ and measure the distance $d$ from the cell boundary where the MN needs to initiate the handoff procedure. Through our analysis, we observe that when the MN's speed is large and traffic load is high, it needs to initiate handoff earlier. By taking the right decision of handoff initiation time we can reduce the handoff failure probability and unnecessary traffic load resulting call termination. Our simulation results show that this approach supports the seamless handoff in Next Generation Wireless Systems (NGWS) effectively.

\section{REFERENCES}

[1] Hye-Soo Kim, Sang Hee Park, Chun-Su Park, Jae Won Kim and SungJea Ko. "Selective Channel Scanning for Fast Handoff in Wireless LAN using Neighbor Graph", July 2004.

[2] Hongqiang Zhai, Xiang Chen, and Yuguang Fang. "How well can the IEEE 802.11 wireless lan support quality of service?" IEEE Transactions on Wireless Communications, 4(6):3084-3094, December 2005.

[3] Yi-Bing Lin Imrich Chalmatc, "Wireless and Mobile Network Architectures," pp. 17.

[4] AKYILDIZ, I. F., XIE, J., and MOHANTY, S., "A survey on mobility management in next generation all-IP based wireless systems," IEEE Wireless Communications, vol. 11, no. 4, pp. 16-28, 2004.

[5] STEMM, M. and KATZ, R. H., "Vertical handoffs in wireless overlay networks," ACM/Springer Journal of Mobile Networks and Applications(MONET), vol. 3, no. 4, pp. 335-350, 1998.

[6] Jaeyoung Choi Student Member, IEEE, Taekyoung Kwon \& Yanghee Choi, Senior Member IEEE, Sangheon Pack Member, , IEEE,Fast Handoff Support in IEEE 802.11 Wireless Networks.

[7] Arunesh Mishra, Minho Shin \& William Arbaugh, An Empirical Analysis of the IEEE 802.11 MAC Layer Handoff Process. [Anshuman Singh Rawat \& Henning Schulzrinne Reducing MAC Layer Handoff Latency in IEEE 802.11 Wireless LANs.

[8] Sangho Shin, Andrea G. Forte, Context Caching using Neighbor Graphs for Fast Handoffs in a Wireless Network.

[9] S. Park and Y. Choi Pre-authenticated fast handoff in a public wireless LAN based on IEEE802.1x mode IFIP TC6 Personal Wireless Communications. Singapore, October 2002.

[10] Jin Teng, Changqing Xu, Weijia Jia, Dong Xuan, D-scan: Enabling Fast and Smooth Handoffs in AP-dense802.11 Wireless Networks.
[11] Chien-Chao Tseng, K-H Chi, M-D Hsieh \& H-H Chang, Location-based Fast Handoff for 802.11 Networks. IEEE COMMUNICATIONS LETTERS, VOL9, NO 4 April 2005.

[12] S.Mohanty and I.F. Akyildiz,'A Cross-Layer(Layer 2+3) Handoff Management Protocol for Next-Generation Wireless Systems',IEEE Transactions On Mobile Computing,Vol-5,No-10 OCT 2006.

[13] Q. Zhang, C. Guo, Z. Guo and W. Zhu, Wireless and Networking Group, Microsoft Research Asia," Efficient Mobility Management for Vertical Handoff between WWAN and WLAN" IEEE,communications Magazine,2003.

[14] I.F. Akyildiz, J. Xie, and S. Mohanty, "A Survey on Mobility Management in Next Generation All-IP Based Wireless Systems," IEEE Wireless Comm., vol. 11, no. 4, pp. 16-28, Aug. 2004.

[15] I.F. Akyildiz, S. Mohanty, and J. Xie, "A Ubiquitous Mobile Communication Architecture for Next Generation Heterogeneous Wireless Systems," IEEE Radio Comm. Magazine, vol. 43, no. 6, pp. S29-S36, June 2005.

[16] Brannstorm, R. Kodikara, E.R. Ahlund, C. Zaslavsky, A Mobility Management for multiple diverse applications in heterogeneous wireless networks, Consumer Communications and Networking Conference, 2006. CCNC 2006. $3^{\text {rd }}$ IEEE.

[17] K. Ayyappan and P. Dananjayan, RSS Measurement for Vertical Hnadoff in Heterogeneous Network, Journal of Theoritical and Applied Information

[18] Leu, A. E, Mark, B. L., "Local Averaging for Fast Handoffs in Cellular Networks" IEEE Transactions On Wireless Communications, Vol. 6, No.3, March 2007, pp. 866 - 874.

[19] Y. Kim, K. Lee and Y. Chin, "Analysis of Multi-level Threshold Handoff Algorithm", Global Telecommunications Conference(GLOBECOM'96), vol. 2, 1996, pp. 1141-1145.

[20] S. BEN OUBIRA, M. FRIKHA, S. TABBANE,"Handoff Management In 4G Networks", IWCMC '09, June 21-24, 2009, Leipzig, Germany.ACM 978-1-60558-569-7/09/06.

[21] Y. Kim, K. Lee and Y. Chin, "Analysis of Multi-level Threshold Handoff Algorithm", Global Telecommunications Conference(GLOBECOM'96), vol. 2, 1996, pp. 1141-1145.

[22] D. P. Agarwal and Q. A. Zeng,' Introduction to Mobile and Wireless Systems', chapter 3.

[23] Distance measurement

[24] Cell sectoring

[25] Prescanning

[26] Carrier to interference ratio

[27] Curve fitting

[28] Cahnnel allocation scheme

[29] Introducing new cell coverage area.

[30] Vector analysis method

[31] Tangent analysis method

\section{AUTHORS PROFILE}

Suman Kumar Sikdar is currently pursuing his $\mathrm{PhD}$ at Kalyani University . He Completed his M.Tech in CSE from jadavpur University in 2011 and B-Tech in Computer Science \& Engineering from Mursidabad College of engineering and technology under West Bengal University of Technology in 2007. His research interest includes wireless communication and satellite communication. Email:sikdersuman@gmail.com

Uttam Kumar kundu Completed his M.Tech in ECE from WBUT in 2010 and B-Tech in E.I.E from JIS College of engineering and technology under West Bengal University of Technology in 2006. His research interest includes wireless communication and satellite communication. Email:kunduuttam@yahoo.co.in

Debabrata Sarddar (Asst. Professor in Kalyani University) is currently pursuing his $\mathrm{PhD}$ at Jadavpur University. He completed his M.Tech in Computer Science \& Engineering from DAVV, Indore in 2006, and his B.Tech in Computer Science \& Engineering from Regional Engineering College(NIT), Durgapur in 2001. His research interest includes wireless and mobile communication Email:dsarddar@ $@$ rediffmail.com. 